\title{
Geant4 simulations of neutron production in a thorium fuelled Accelerator Driven Subcritical Reactors
}

\section{David Sangcheol Lee ${ }^{1}$}

International Institute for Accelerator Applications, University of Huddersfield

IIAA, University of Huddersfield, Queensgate, Huddersfield, West Yorkshire, UK HD1 3DH

E-mail: Sangcheol.lee@hud.ac.uk

\section{Robert Cywinski}

International Institute for Accelerator Applications, University of Huddersfield

IIAA, University of Huddersfield, Queensgate, Huddersfield, West Yorkshire, UK HD1 3DH

E-mail: R. Cywinski@hud.ac.uk

\section{Cristian Bungau}

International Institute for Accelerator Applications, University of Huddersfield

IIAA, University of Huddersfield, Queensgate, Huddersfield, West Yorkshire, UK HD1 3DH

E-mail: C.Bungaulhud.ac.uk

\section{Rebecca Seviour}

International Institute for Accelerator Applications, University of Huddersfield

IIAA, University of Huddersfield, Queensgate, Huddersfield, West Yorkshire, UK HD1 3DH

E-mail: R.Seviour@hud.ac.uk

Spallation taken from the middle English spalle, is an efficient process for producing an intense neutron flux suitable for exploitation in Accelerator Driven Subcritical Reactors (ADSRs) for energy production and the transmutation. In order to assess spallation driven fission and transmutation we have simulated proton induced neutron production using GEANT4. Benchmarking our simulations against experimental neutron spectra produced from a thick lead target bombarded with 0.5 and $1.5 \mathrm{GeV}$ protons. The Bertini and INCL models available in GEANT4, coupled with the high precision (HP) neutron model, are found to reproduce the published experimental data. Given the confidence in the GEANT4 simulations provided by this benchmarking we then proceed to study neutron production in an ADSR using Thorium in a geometry similar to that of the proposed Belgian MYRRHA project with $0.6 \mathrm{GeV}$ proton beam energy that proposed by SCKCEN.

4th Workshop on ADS and thorium

31 August - 2 September 2016

University of Huddersfield, England

${ }^{1}$ Speaker 


\section{Introduction}

Spallation is a reliable technique for the production of high intensity neutron fluxes. These sources use high intensity proton beams with energies of typically 1-2 GeV, optimised target producing neutrons. In this paper we use a Monte-Carlo based transport simulation to model spallation reactions. GEANT4, a Monte-Carlo based transport code developed by CERN, provides an extensive set of hadronic physics models for energies up to $10-15 \mathrm{GeV}$, both for the intranuclear cascade region and for modelling of evaporation [1]. GEANT4 codes records particle histories and probabilistic interactions, with the simulated events determined by sampling associated probability distributions. In this paper we evaluate the suitability of GEANT4 to simulate the spallation process, by benchmarking GEANT4 results against published experimental observations [2]. Having confirmed the suitability of GEANT4 to model the spallation process we can then use GEANT4 to optimise the target geometry for optimal neutron yield. We also present the neutron energy spectrum resulting from embedding a spallation target within an assembly of thorium fuel assemblies, reflectors and shielding in a geometry similar to that of the proposed ADSR project MYRRHA [3,4]. The MYRRHA design uses a $600 \mathrm{MeV}$ proton linear accelerator, a spallation target and a multiplying core with uranium based MOX fuel where the core area is cooled by liquid lead-bismuth [5], which also provides the spallation target material [6]. A schematic of MYRRHA reactor core is shown in Figure 3

\section{Geant4 benchmarking study}

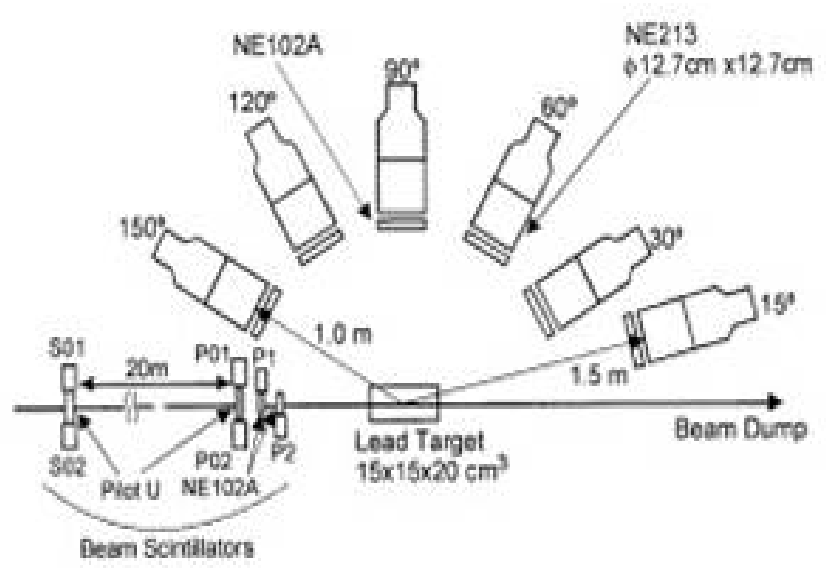

Figure 1: Experimental arrangement, taken from [2]. 
For our initial simulations, we choose a configuration similar to that used in the experiments [2]. The experimental setup of [2] is shown in Figure 1 where proton beam incident onto a lead spallation tater. The spallation target was placed at the centre of a simulated space with detectors placed at 15, 30, 60, 90, 120 and 150 degrees with respect to the target and incoming proton beam, at a distance of 1 metre, except the $15^{\circ}$ detector which was placed at 1.5 metres [2]. The area of each detector was set to $12.7 \times 12.7 \mathrm{~cm}^{2}$ the same size as the detector in [2].

GEANT4 provides numerous hadronic physics models describing various particle interactions. For the work presented in this paper, the following GEANT4 physics list were used. QGSP_BERT_HP which uses the Bertini intranuclear cascade model to describe the inelastic interactions of protons and neutrons, QGSP_BIC_HP which uses the theory based Binary Cascade model capable of simulating secondary particle production by creating more physical path of particle interaction [7], and QGSP_INCLXX_HP which uses the latest developed Liège model that has improved interpretation of pre-equilibrium and other particle interactions [8]. Coupled with a high precision (HP) neutron model using data for neutron cross-sections below $20 \mathrm{MeV}$ [4].

\subsection{Result of the simulation for benchmarking}

Figure 2a show the neutron spectra (neutrons/ incident proton / steradians in $1 \mathrm{MeV}$ bin size) at various projectile angles between 0 and $100 \mathrm{MeV}$ neutron energy. It can be seen that there is a good agreement between the GEANT4 results and the experimental data throughout the whole spectrum. More specifically, the green line shown in Figure 2a which represents the results produced by QGSP_INCLXX_HP physics list show closest to the experimental results. While this physics produced most accurate results to the experimental result throughout the entire neutron energy range, the graphs shows that INCL based physics list produced result significantly close with the experimental result in the range between $0-10 \mathrm{MeV}$ neutron energy.

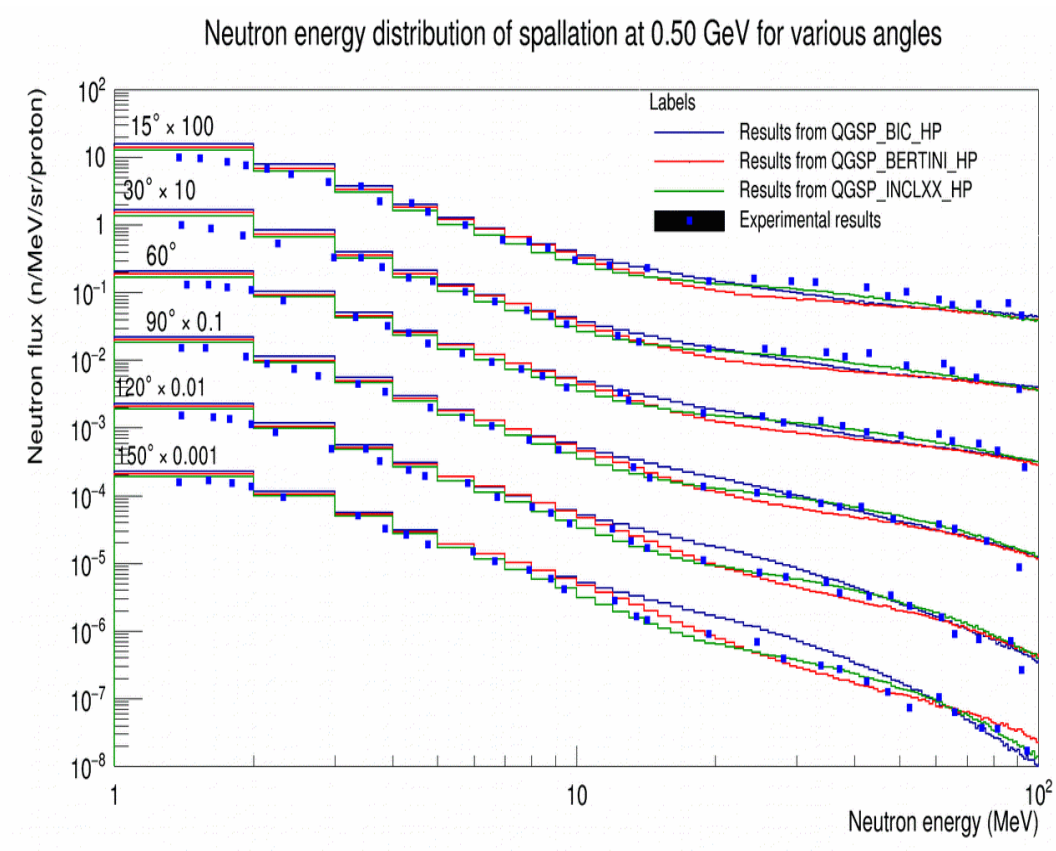

Figure 2a: Neutron flux at $0.5 \mathrm{GeV}$ proton beam energy using GEANT4 at the neutron energy between 0 and $100 \mathrm{MeV}$ with the experimental data taken from [2] 


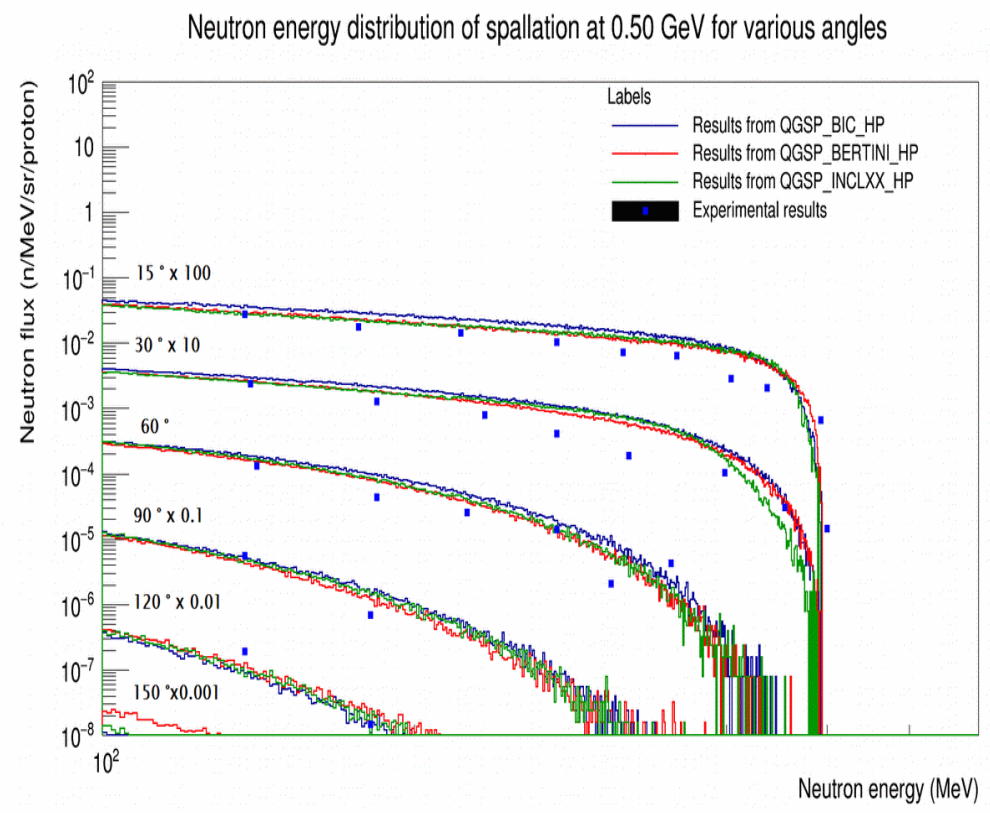

Figure 2b: Neutron flux at $0.5 \mathrm{GeV}$ proton beam energy in the range of neutron energy between 100 and $600 \mathrm{MeV}$ using GEANT4 with the experimental data taken from [2]

In Figure 2a, the least accurate result from INCL based physics list to the experimental result is found to be 0.0488 , which is $4.88 \%$ error at neutron energy $91 \mathrm{MeV}$ in $120^{\circ}$. This error estimate supports that Geant4 is capable of producing reliable results for spallation reaction. On the other hand, The Figure $2 b$ shows a decrease in the accuracy of Geant 4 to experimental results. However, the curves in each angle follows the trend of experimental results. The least accurate point by QGSP_INCLXX_HP in Figure 2b is found as, $3.11 \times 10^{-3}$ which is $5.99 \%$ error at neutron energy $437 \mathrm{MeV}$ in $15^{\circ}$.

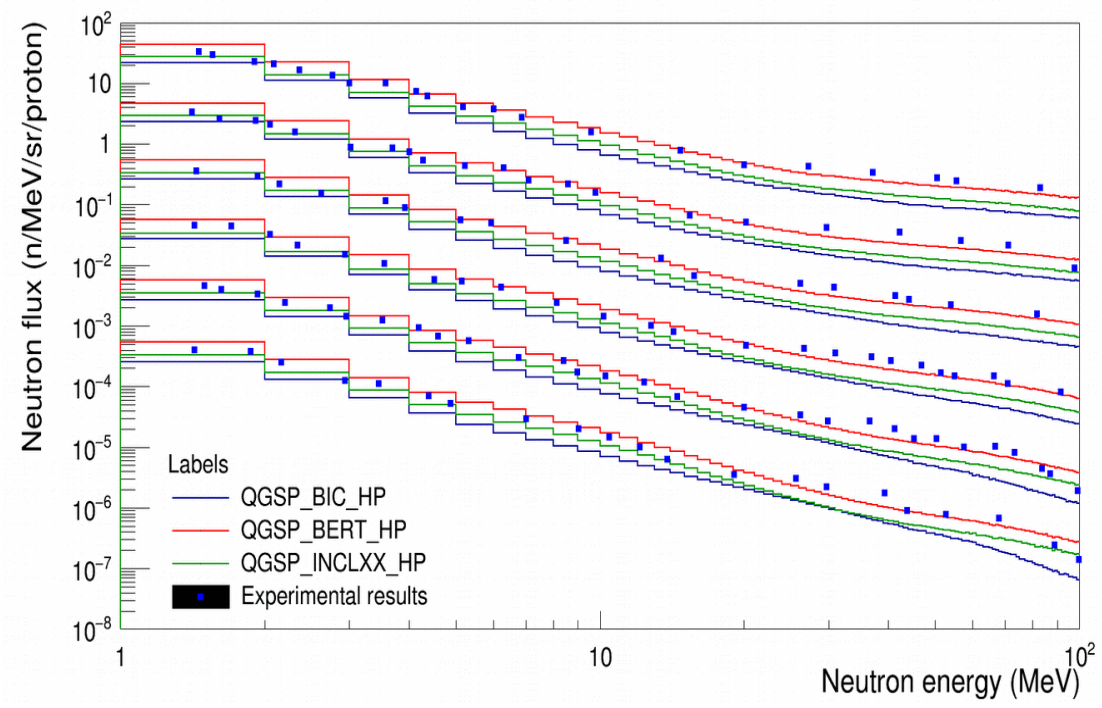

Figure 2c: Neutron flux at $1.5 \mathrm{GeV}$ proton beam energy in the range of neutron energy between 0 and $100 \mathrm{MeV}$ using GEANT4 with the experimental data taken from [2] 


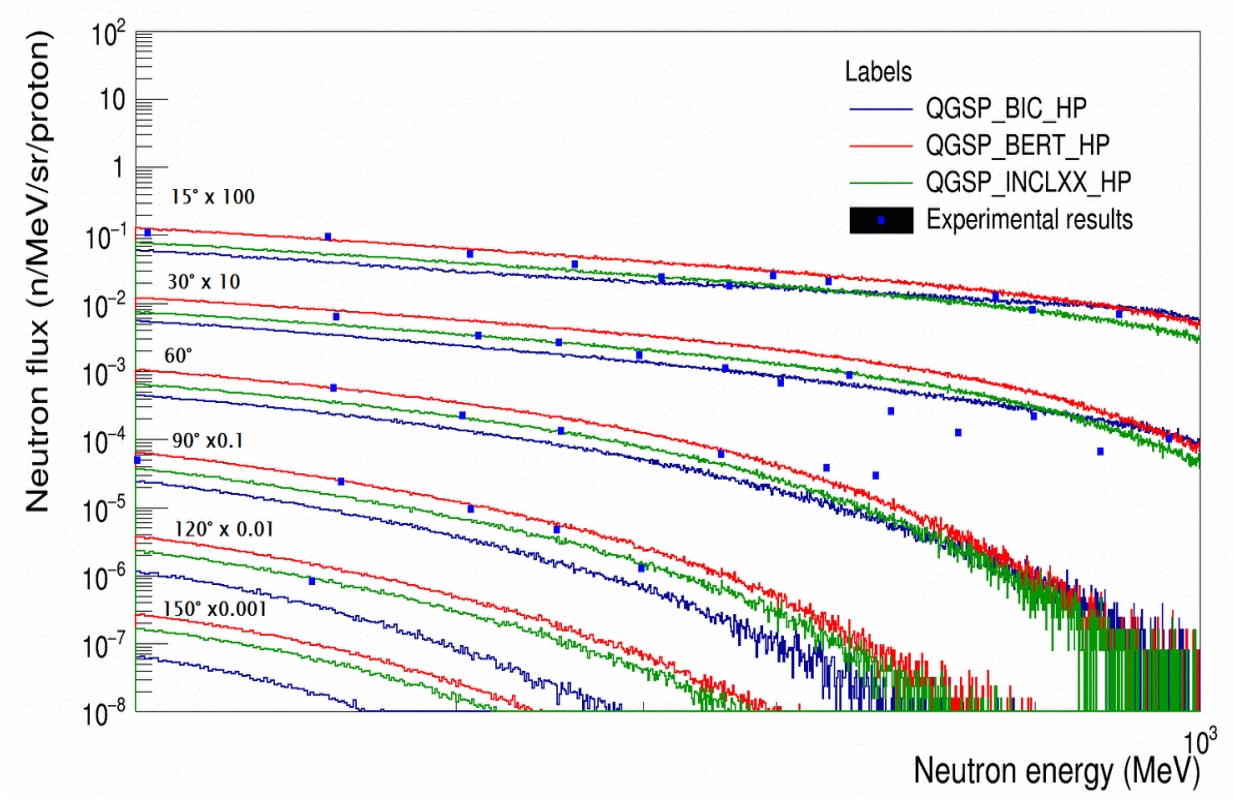

Figure 2d: Neutron flux at $1.5 \mathrm{GeV}$ proton beam energy in the range of neutron energy between 100 and $1000 \mathrm{MeV}$ using GEANT4 with experimental data taken from [2]

Similarly, the Geant 4 results with experimental results at $1.5 \mathrm{GeV}$ beam energy shown in Figure $2 \mathrm{c}$ and $2 \mathrm{~d}$ indicate similar trend as the results shown in Figure $2 \mathrm{a}$ and $2 \mathrm{~b}$. Initially, it was anticipated that there would be a significant difference between simulation and experiment due to the difficulty measuring of high energised neutrons in both experiment and simulation methods. It can be seen from that Geant 4 produces fairly accurate results to the experiment results as shown in Figure 2c. The least accurate point is found to be at neutron energy of $55 \mathrm{MeV}$ in $30^{\circ}$ with the difference of $3.89 \times 10^{-6}$ which is $6.64 \%$. The accuracy of Geant 4 simulation result to the experiment is decreasing as the neutron energy spectrum increases. In Figure $2 d$, it shows that the Geant4 results get fragmented in high energy range due to the low number of neutrons presented. The difference between Geant4 result and the experimental results of neutron energy of $697 \mathrm{MeV}$ in $30^{\circ}$ was $8.61 \times 10^{-5}$ which is $27.97 \%$. As previously mentioned, the accuracy of measuring high energy neutron is low in both experimental and simulation methods. Hence, the reliability for measuring such high neutron energies can not be certain. In overall, of the three physics models we have used, the QGSP_INCLXX_HP physics list produced the closest results to the experimental results, followed by the Bertini, whilst the Binary Intra-nuclear Cascade (BIC) proved to be the least accurate. The results from INCL at $0.5 \mathrm{GeV}$ proton beam energy showed a match to the experimental results at every angle. The Bertini model also produced accurate results at between 0 and $10 \mathrm{MeV}$ neutron energy (see Figure 2a). At $1.5 \mathrm{GeV}$, INCL produced the most accurate outcome in relation to the experimental results in the neutron energy range between $0-10 \mathrm{MeV}$ and 100-1000 MeV (see Figure $2 \mathrm{~d})$. However the Bertini model produced better results than the INCL at the range between 10-100 MeV (see Figure 2c). The above simulations indicate that GEANT4 
is capable of modelling spallation neutron reactions in an energy regime useful for exploitation in an ADSR.

\section{Neutron energy distribution in different regions with MYRRHA's core configuration}

For the paper we consider a reactor geometry similar to MYRRHA where we have replaced the Uranium based reactor fuel by fertile thorium. Thorium is currently attracting interest as both a potential fuel and a matrix for transmutation of nuclear waste. In order to understand the effect of the reflector, in this paper the simulations were carried out with the shielding assemblies removed. The configured geometry of core assembly with thorium fuel is shown in figure 3 and its specification in table 1. In figure 3, each assembly area has different colours corresponding to those composed materials such as blue is spallation target, yellow is thorium fuel, green as reflector and cyan as inner shielding.

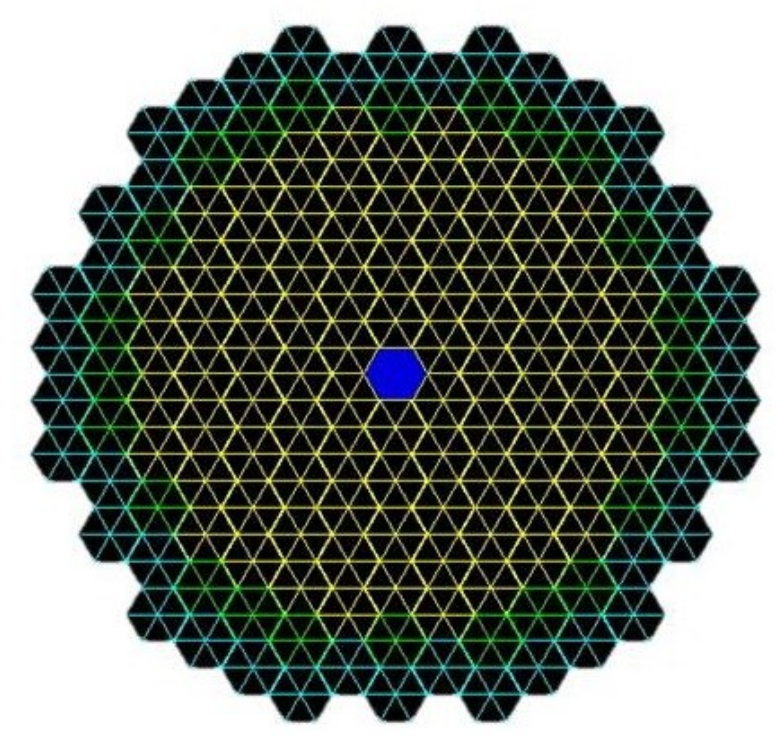

Figure 3: Geant4's MYRRHA core configuration (Blue: spallation target, Yellow: thorium fuel, Green: reflectors, Cyan: inner shielding)

\begin{tabular}{|c|c|c|c|}
\hline Area & Material & Size $\left(\mathrm{mm}^{2}\right)$ & $\begin{array}{c}\text { Number of core } \\
\text { units }\end{array}$ \\
\hline $\begin{array}{c}\text { Spallation } \\
\text { target }\end{array}$ & $\mathrm{PbBi}$ & $97.55 \times 300$ & 1 \\
\hline Fuel & Thorium & $97.55 \times 1400$ & 76 \\
\hline Reflectors & $\mathrm{PbBi}$ & $97.55 \times 1400$ & 36 \\
\hline Shielding & $\begin{array}{l}\mathrm{Y}_{2} \mathrm{O}_{3} \\
\mathrm{ZrO}_{2} \\
\mathrm{Zr}_{2} \mathrm{O}_{3}\end{array}$ & $97.55 \times 1400$ & 42 \\
\hline
\end{tabular}

Table 1: Core specification used in the simulation 
The results of the GEANT4 simulations, figures $3 a, 3 b$ and $3 c$, show the energy spectra of neutrons travelling from one region to another in the core. The distance of each annular region from the centre of core is set as the size of a fuel assembly (i.e. region-1 $97.55 \mathrm{~cm}$, region-2 $195.1 \mathrm{~cm}$ etc.). In figure $3 \mathrm{a}$, it can be seen that the number of outer going neutrons per proton for fuel region 1 to 2(red line) is higher than the number of neutrons from target to region 1 (blue line). This result indicated that the first fuel region contributed to neutron production significantly. On the other hand, figure $3 \mathrm{~b}$ shows differently that the difference of the results between the target to region 1 and region 1 to region 2 is very small which indicates that the production of high energy neutrons is not increased significantly by thorium fuel area.

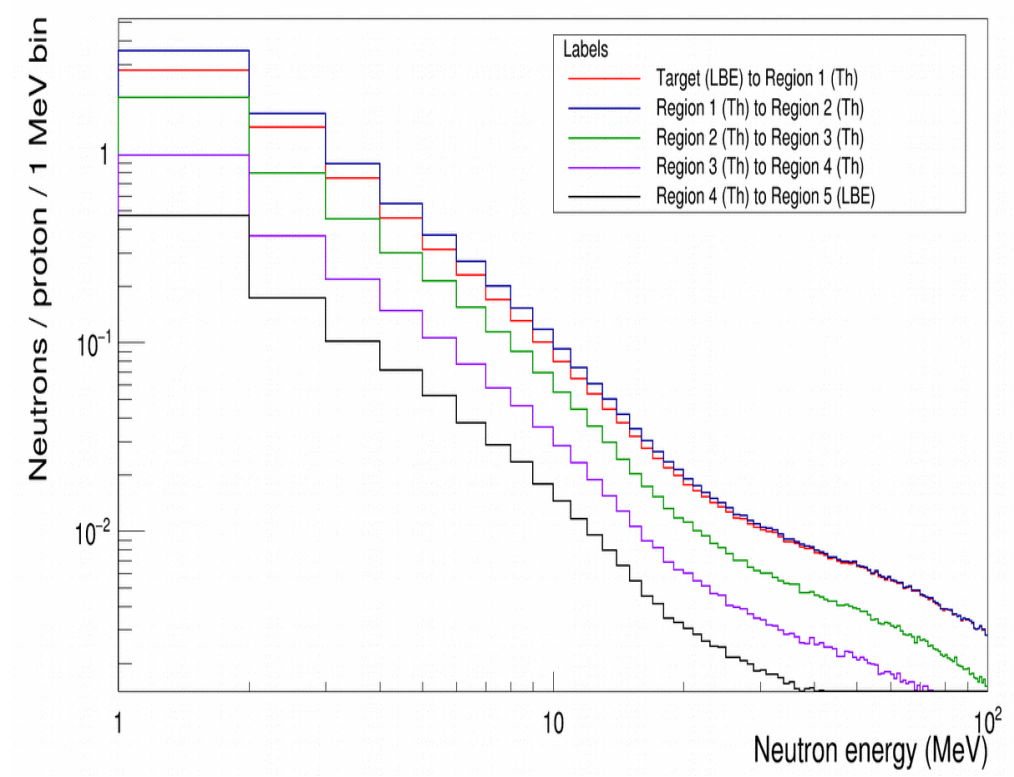

Figure 3a: Number of outer going neutrons into each region in the core as the function of neutron energv hetween 0 and $100 \mathrm{MeV}$

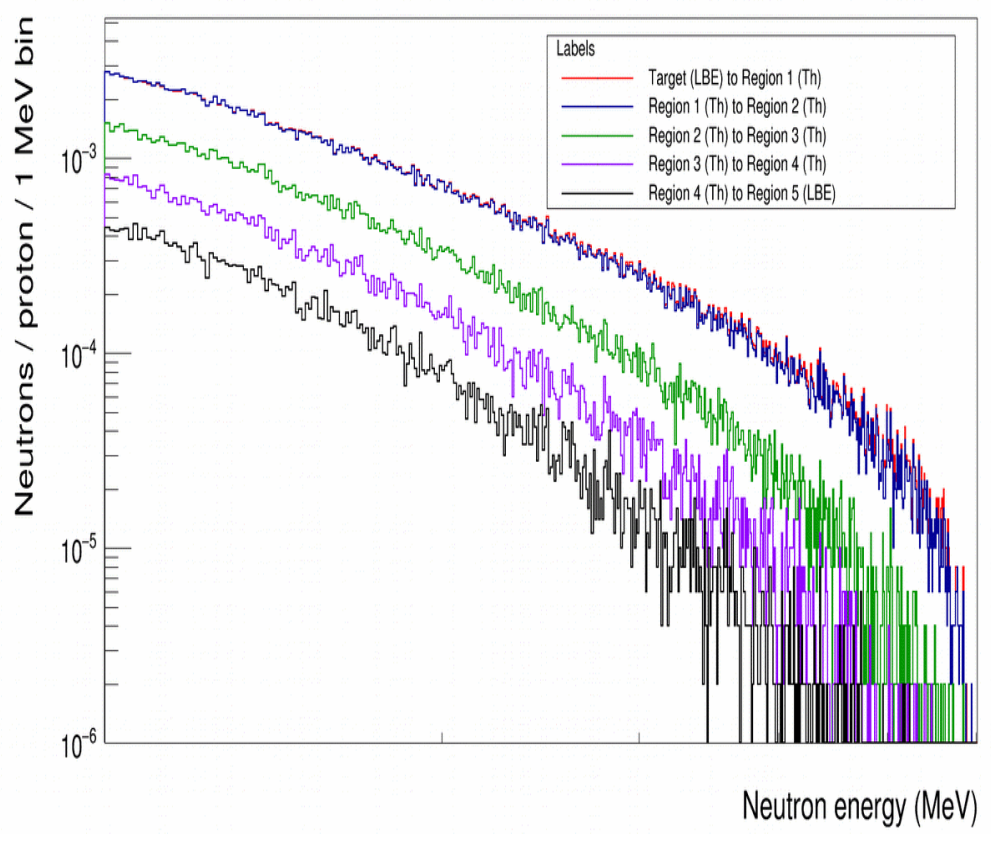

Figure 3b: Number of outer going neutrons into each region in the core as a function of neutron energy between 100 and $1000 \mathrm{MeV}$ 


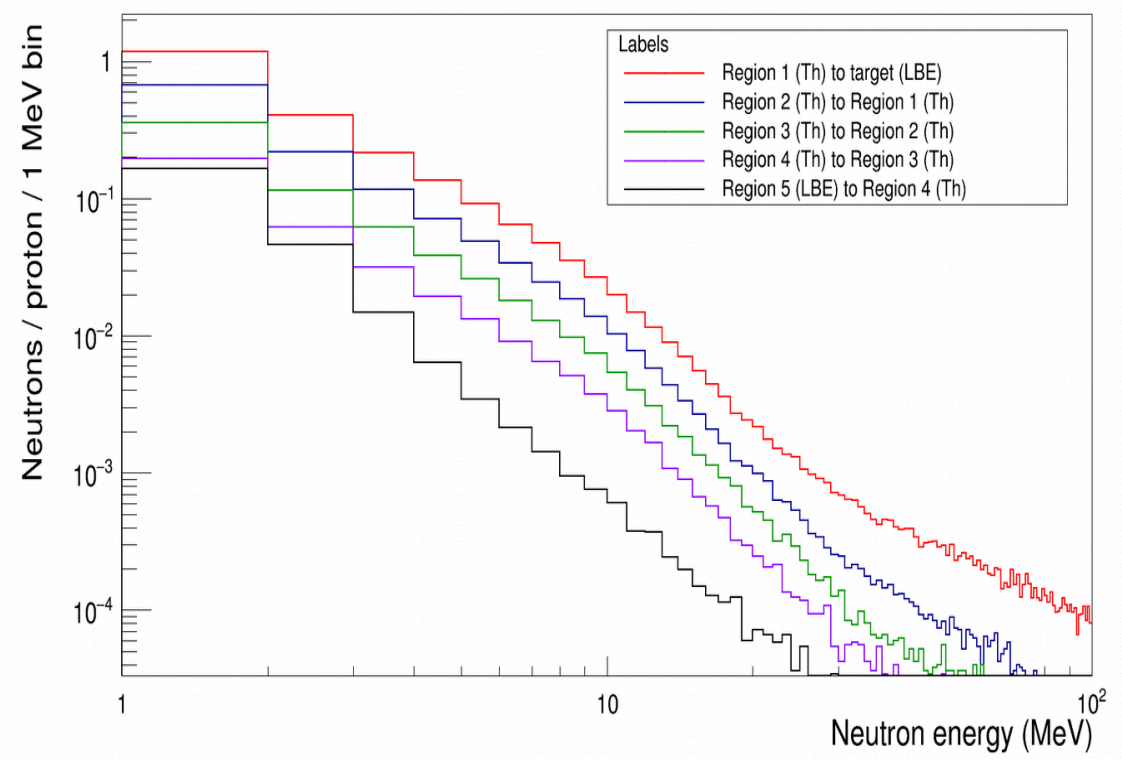

Figure 3c: Number of neutron back-scattered into each region in the core as a function of neutron energy between 0 and $100 \mathrm{MeV}$

In figure $3 \mathrm{c}$, the area that neutrons are most travelling backward is the area from region 1(composed with thorium) to the spallation target(composed with lead bismuth eutectic) The area where neutrons travelling from the region 1 to the spallation target is the first region placed after spallation target. Hence, it is expected to get the results of having the high rate of neutrons back-scattered in the closest area from the centre of core.

\subsection{The energy distribution of neutron escaping from the core with reflectors.}

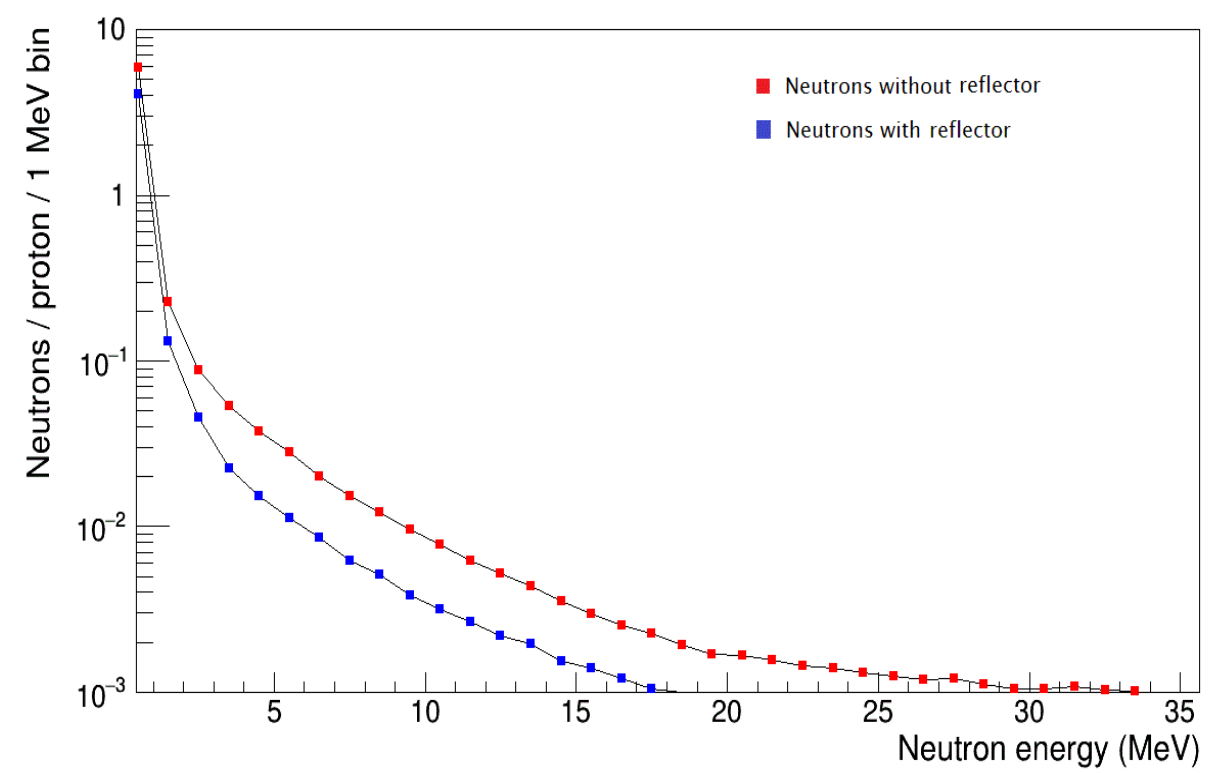

Figure 4: Number of neutrons escaped from the core as a function of neutron energy taken from [10]

Comparing neutron energy spectra between the reflector and the non-reflector as shown in Figure 7, the reflectors decrease the number of neutrons per proton in high neutron 
energy. The figure also indicates that the maximum neutron energy of the non-reflector result was recorded at $33.5 \mathrm{MeV}$ while the highest neutron energy for the reflector was at $17.5 \mathrm{MeV}$. Regarding the number of neutrons escaping from the core per proton, there is no significant difference between the reflector and the non-reflector at the neutron energy range of 0-2 $\mathrm{MeV}$. This result indicates that the nuclei of the reflector material (i.e. LBE) were not effective for stopping neutrons escaping from the reflector.

\subsection{The energy distribution of neutron escaping from the core with inner shielding.}

The core consists of many areas of shielding in the structure to increase neutron flux and block radiation emitting from the core. The original MYRRHA core specification indicated that there is an 'outer dummy' area in placed after the reflector region for shielding purpose. Due to the lack of information of the composition of YZrO listed in [9], three different materials close to $\mathrm{YZrO}$ were tested to investigate the effect of blocking neutrons escaping from the core. In this text, this area of shielding is named as 'inner shielding' to express the purpose of the area in the core.

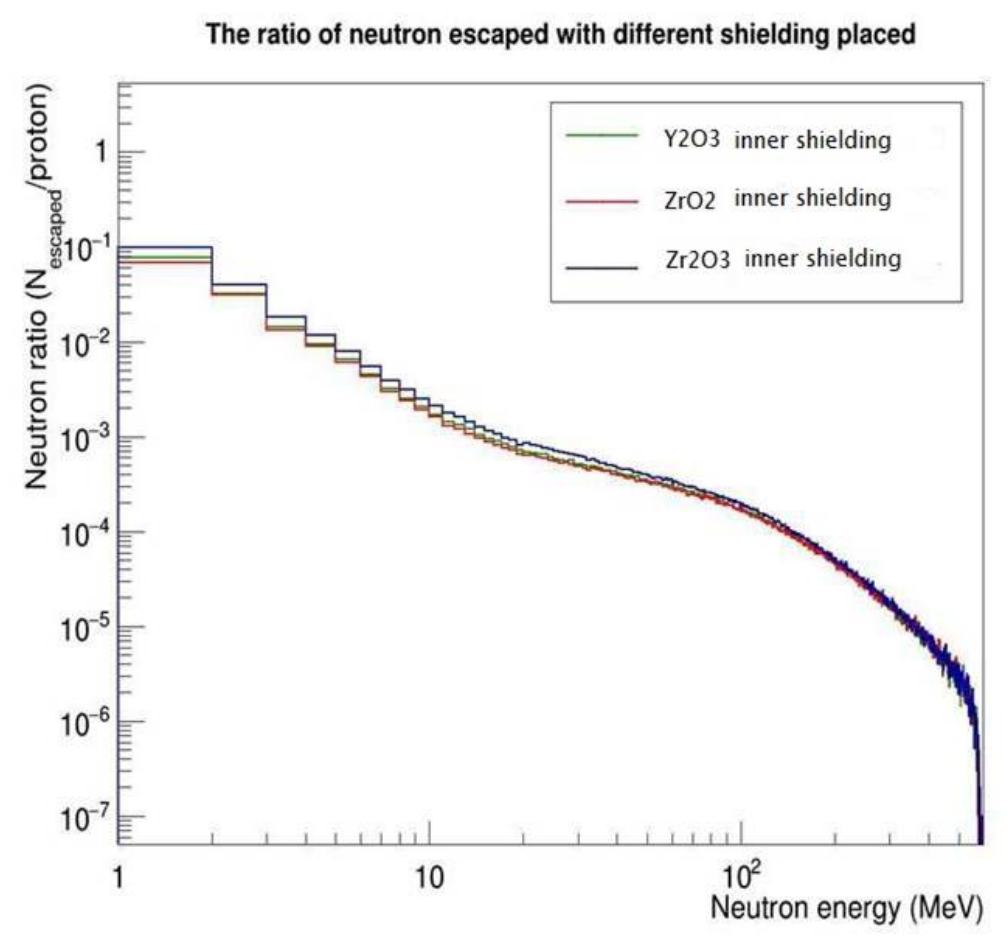

Figure 5: Energy distribution of neutrons escaping from the core with different inner shielding as a function of neutron energy

Comparing the neutron energy spectra between the three materials shown in Figure 8, the number of neutrons escaping per incident proton is similar in all three materials, especially for high neutron energies. Figure 8 also indicates that the highest rate of neutrons blocking for neutron energies between 0 and $2 \mathrm{MeV}$ was achieved by $\mathrm{ZrO}_{2}$. However the difference between $\mathrm{ZrO}_{2}$ with other two candidate $\left(\mathrm{Y}_{2} \mathrm{O}_{3}\right.$ and $\left.\mathrm{Zr}_{2} \mathrm{O}_{3}\right)$ is very small. This result indicates that the rate of neutron escaping from the core may be varied when the further shielding area is in placed. 


\section{Conclusion}

In this paper, we have shown that GEANT4 simulations of spallation neutron production agree well with experimental data. The QGSP_INCLXX_HP physics list appears to reproduce the experimental results most closely.

Our simulation of a thorium fuelled MYRRHA type reactor show that reflectors have a significant effect reducing the neutrons escaping from the core. However, the number of neutrons per proton escaping is high. This suggests that extra shielding is necessary for increasing neutron flux inside the core which reflects the rate of thorium-uranium conversion.

The inner shielding area composed of three different materials suggested that all three materials had similar rates of reducing neutrons escaping from the core. This suggested that the result may be varied when outer shielding area is in place.

\section{Reference}

[1] C. Bungau, R. Cywinski, and R. Barlow, "Neutron spallation studies for an accelerator driven subcritical reactor," in Particle Accelerator Conference, 2009.

[2] S. Meigo, H. Takada, S. Chiba, T. Nakamoto, K. Ishibashi, N. Matsufuji, K. Maehata, N. Shigyo, Y. Watanabe, and M. Numajiri, "Measurements of neutron spectra produced from a thick lead target bombarded with $0.5-$ and $1.5-\mathrm{GeV}$ protons," Nucl. Instruments Methods Phys. Res. Sect. A Accel. Spectrometers, Detect. Assoc. Equip., vol. 431, no. 3, pp. 521-530, Jul. 1999.

[3] M. Sarotto, "MYRRHA-FASTEF FA / core design," in international Workshop on Innovative Nuclear Reactors cooled by HLM, 2012.

[4] S. Agostinelli, J. Allison, K. Amako, and Et.al, "GEANT4 - A simulation toolkit," Nucl. Instruments Methods Phys. Res. Sect. A Accel. Spectrometers, Detect. Assoc. Equip., vol. 506, no. 3, pp. 250-303, 2003.

[5] H. Aït Abderrahim, "MYRRHA An innovative and unique irradiation research facility," in Tenth International Topical Meeting on Nuclear Applications of Accelerators (AccApp'11), 2011.

[6] SCK•CEN, “Applications catalogue of MYRRHA.” [Online]. Available: http://myrrha.sckcen.be/en/MYRRHA/Applications. [Accessed: 21-Apr-2016].

[7] D. H. Wright, "An Overview of Geant4 Hadronic Physics Improvements," in Joint International Conference on Supercomputing in Nuclear Applications and Monte Carlo (SNA + MC2010), 2010.

[8] J. Yarba, "Recent Developments and Validation of Geant4 Hadronic Physics," J. Phys. Conf. Ser., vol. 396, no. 2, p. 22060, Dec. 2012. 
[9] P. Baeten, M. Schyns, R. Fernandez, D. De Bruyn, and G. Van den Eynde, "MYRRHA : A multi-purpose nuclear research facility," EPJ Web Conf., vol. 79, no. 3001, Nov. 2014.

[10] S. C. Lee, C. Bungau, and R. Cywinski, "Geant4 simulations of proton-induced spallation for applications in ADSR systems," in Proceedings of IPAC 2016, Busan, Korea, 2016, vol. 08 Applications for Accelerators, pp. 1943-1945. 